\title{
Determinants of Knowledge and Attitude Towards Breastfeeding Using Validated Instruments in Pregnant Women From a Rural Setting in Ethiopia
}

Misra Abdulahi Ahmed ( $\square$ misra_ab@yahoo.com )

Universitetet i Oslo https://orcid.org/0000-0001-5524-2910

Atle Fretheim

University of Oslo: Universitetet i Oslo

Alemayehu Argaw

Jimma University

Jeanette $\mathrm{H}$. Magnus

University of Oslo: Universitetet i Oslo

\section{Research}

Keywords: breastfeeding, knowledge, attitude, determinants, rural Ethiopia

Posted Date: January 11th, 2021

DOI: https://doi.org/10.21203/rs.3.rs-141931/v1

License: (c) (i) This work is licensed under a Creative Commons Attribution 4.0 International License. Read Full License 


\section{Abstract}

\section{Background}

Understanding the important underlying determinants of maternal knowledge and attitude towards breastfeeding guides the development of context-specific interventions aimed at increasing the rates of optimal breastfeeding practices. However, studies that used validated instruments to assess breastfeeding knowledge and attitude are nonexistent in Ethiopia.

Objective

To assess the level and determinants of breastfeeding knowledge and attitude using validated instruments in pregnant women who participated in breastfeeding education and support intervention in a rural district in Ethiopia.

\section{Methods}

468 pregnant women in their second or third trimester were interviewed at baseline to assess their knowledge and attitude towards breastfeeding practices using locally adapted and validated instruments. We used the Afan-Oromo versions of the Breastfeeding Knowledge Questionnaire (BFKQ-AO) and the lowa Infant Feeding Attitude Scale (IIFAS-AO). Breastfeeding knowledge and attitude scores were standardized based on the distribution of the population and multiple linear regression models were fitted to identify the independent determinants knowledge and attitude.

Results

$52.4 \%$ of the mothers had a high level of knowledge while $60.9 \%$ of the women had a neutral attitude towards breastfeeding. In a multiple linear regression model, the maternal occupation was the only predictor of the overall BFKQ-AO score (0.56 SD; $95 \% \mathrm{Cl}, 1.28,4.59 \mathrm{SD} ; P=0.009)$. Age (0.57 SD; $95 \% \mathrm{Cl}$, $0.24,0.90 \mathrm{SD} ; P=0.001)$, parity $(-0.24 \mathrm{SD} ; 95 \% \mathrm{Cl},-0.47,-0.02 \mathrm{SD} ; P=0.034)$, antenatal care visits (0.41 SD; $95 \% \mathrm{Cl},(0.07,0.74 \mathrm{SD} ; P=0.017)$ and the BFKQ score (0.08 SD; $95 \% \mathrm{Cl}, 0.06,0.09 \mathrm{SD} ; P<0.000$ ) were predictors of the IIFAS-AO score.

\section{Conclusions}

Although more than half of the respondents had adequate knowledge about breastfeeding, most women had a neutral attitude towards breastfeeding. Occupation of mothers was an independent predictor of breastfeeding knowledge, whereas age, parity, antenatal care visits, and breastfeeding knowledge score were predictors of breastfeeding attitude. Thus, policymakers and managers should address these factors when planning educational interventions on breastfeeding to improve knowledge and attitude thereby improving breastfeeding practices.

\section{Background}


Exclusive breastfeeding (EBF) of infants during the first six months of life is recommended [1], given the several benefits identified for both mother and infant [2-12]. Despite the presence of global recommendations, the practice of EBF substantially lagged behind the level recommended by the World Health Organization, especially in lower and middle-income countries where the burden of suboptimal infant feeding is high [13]. In Ethiopia, early initiation of breastfeeding is $78 \%$ while $58 \%$ of children under 6 months old are exclusively breastfed [14]. Although the current rate of early initiation and EBF are high compared to the global rate, both are below the 2020 national target of the Ethiopian Health Sector Transformation Plan of $90 \%$ and $72 \%$, respectively [15]. Moreover, after birth, the EBF-rate declines rapidly from $74 \%$ between $0-1$ month to $36 \%$ at $4-5$ months [14]. So although the rate of EBF increased from $55 \%$ in 2000 to $60 \%$ in 2016, this increase was neither substantial, nor it is within reach of the Health Sector Transformation Plan target plan.

Large studies undertaken to estimate the prevalence and determinants of EBF practice have identified different socio-demographic and psychosocial factors such as mother's knowledge and attitude [16-18]. For example, positive maternal attitude and good knowledge are pivotal in the process of breastfeeding and regarded as modifiable variables when aimed to improve breastfeeding practices. In turn, other studies have reported that knowledge about the benefits of breastfeeding is significantly associated with breastfeeding attitude; positive maternal attitude toward breastfeeding is a strong predictor of breastfeeding initiation and longer duration than sociodemographic factors [19-25]. In Ethiopia, several studies were conducted to assess knowledge and attitude towards breastfeeding as factors that influenced breastfeeding practice. Yet all the available studies have used non-validated tools and ended up with varied factors across different settings [26-28].

Understanding the predictors of maternal knowledge and attitudes toward breastfeeding through validated instrument guides the development and implementation of public health policy and is likely to improve the evaluation of interventions aimed at increasing rates of breastfeeding. Such studies have the potential to identify the reasons why breastfeeding was discontinued among women in the first six months of their child's life. However, there is a dearth of local data on a range of factors known to influence the mother's knowledge and attitude towards breastfeeding in Ethiopia using validated instruments.

With the aim to improve breastfeeding knowledge, attitude and practices in Manna district, Southwest Ethiopia we conducted a randomized controlled trial in which we provided pregnant women with a breastfeeding education and support intervention (BFESI) that included prenatal breastfeeding education and the provision of postnatal peer support [29]. As one of the aims of the research project was to assess change in the level of knowledge and attitude towards breastfeeding, we sought to measure the baseline level and identify predictors of knowledge and attitude towards breastfeeding among women who enrolled into the BFESI trial. This investigation will be the heralded event comprehensively exposing the predictors of knowledge and attitude towards breastfeeding in an Ethiopian context.

\section{Methods}




\section{Setting and Participants}

This cross-sectional study used data collected between May and August 2017 from the baseline survey of the BFESI, which has previously been reported [29]. The study was conducted in Mana district, the smallest district in Jimma zone, located approximately 30-40 Km from Jimma town. Agriculture is the main form of livelihood in the study community with coffee accounting for $80 \%$ of the main crops produced in the area. It is the most densely populated district in the zone with 308 persons per $\mathrm{Km}^{2}$. The actual population is estimated at 132,358 .

We recruited 468 pregnant women using the Ethiopian community health system, Health Extension Workers antenatal logbook and Women Development Army leaders, to identify the pregnant women. Participants comprised of healthy, pregnant women in their $2^{\text {nd }}$ or $3^{\text {rd }}$ trimester, without severe health complications including any psychiatric illness, who provided consent to participate in the trial and no plan to leave the study area before completion of the BFESI trial. The study protocol was approved by the Institutional Review Boards of Jimma University, and the ethical review board of Oromia Regional Health Bureau. We obtained written consent from eligible participants after providing an information session detailing the study, voluntary participation, and study withdrawal options.

\section{Data collection}

Ten nurses who were fluent in the local language were trained for 2 days carried out data collection. Data were collected through a structured face-to-face interview after obtaining written consent from study participants. Maternal knowledge and attitude towards breastfeeding were assessed using the Afan Oromo versions of the Breastfeeding Knowledge Questionnaire (BFKQ-AO) and the IOWA Infant Feeding Attitude Scale (IIFAS-AO), which have been locally adapted and validated in the same population [30]. The BFKQ-AO consists of 34 items asking about various optimal breastfeeding practices, with responses coded as correct or incorrect. We used a cut-off of $\geq$ or $<$ the median to categorize knowledge level as the breastfeeding knowledge questionnaire we adopted from Malaysia does not have a cut-off point for an optimal knowledge level. Accordingly, all mothers who scored $\geq$ the median in the knowledge test were considered as having a high level of knowledge and those scoring below the median were considered as having a low level of knowledge. The IIFAS-AO consists of 17 items, with a 5-point Likert scale, rating maternal attitude towards breastfeeding, and total possible scores range from 17 to 85 with a higher score reflecting positive attitude. Attitude toward breastfeeding was categorized into three as follows: 1 ) positive to breastfeeding (IIFAS score 70-85), 2) neutral (IIFAS score 49-69), and 3) positive to formula feeding (IIFAS score 17-48) [31]. Furthermore, data on potential determinants of knowledge and attitude have been gathered such as sociodemographic factors, including maternal age, level of education, number of children, household wealth and food security status, and maternal factors, including parity, ANC visits, past obstetric and breastfeeding history.

\section{Statistical analysis}


Double data entry was performed using EpiData (version 3.1) and all statistical analyses were done using Stata version 13.1 (StataCorp LLC: College Station, TX, USA). Data were summarized using frequencies and percentages. In the first stage of the analysis, we evaluated bivariate associations between potential predictors and the study outcomes breastfeeding knowledge and attitude score to determine candidate predictors for the subsequent multiple linear regression models. In the second stage, based on the result of bivariate association, we fitted multiple linear regression models assessing the independent predictors of breastfeeding knowledge and attitude scores. We applied a robust variance estimation to take into account clustering of subjects by study sub-districts. Knowledge and attitude scores were standardized based on the distribution of our data and thus, results are expressed as standardized regression coefficients and $95 \%$ Cis. Models were evaluated for potential multi-collinearity problems using the variance inflation factor with values less than ten considered acceptable and model goodness of fit was assessed using adjusted $\mathrm{R}^{2}$ values. All tests were two-tailed, and statistically significant association was considered at a $\mathrm{p}$-value $<0.05$.

\section{Results}

The characteristics of 468 pregnant women are presented in Table 1. Most women were in the age group of 20-34 (83.3\%), illiterate (74.6\%), housewives/farmers (93.8\%), and lived in a food-insecure household $(58.9 \%)$. Three hundred and eighty-two (81.6\%) of the enrolled women were multiparous of which $96.8 \%$ had a history of breastfeeding. The majority $(88 \%)$ of the women had at least one ANC visit, but only $7.28 \%$ of them had $\geq 4$ antenatal care visits (Table 1 ). 
Table 1

Characteristics of participants $(\mathrm{N}=468)$.

\begin{tabular}{|ll|}
\hline Variable & No (\%) \\
\hline Age & $50(10.68)$ \\
\hline $15-19$ & $390(83.33)$ \\
\hline $20-34$ & $28(5.98)$ \\
\hline$\geq 35$ & \\
\hline Educational status & $349(74.6)$ \\
\hline Illiterate & $90(19.2)$ \\
\hline Primary school & $29(6.2)$ \\
\hline Secondary school & \\
\hline Wealth tertiles & $156(33.33)$ \\
\hline Lowest & $156(33.33)$ \\
\hline Middle & $156(33.33)$ \\
\hline Highest & \\
\hline Maternal occupation & $370(96.8)$ \\
\hline Housewife/Farmer & $30(6.40)$ \\
\hline Parity & \\
\hline Multiparous & \\
\hline Household food security status & \\
\hline Food insecure & \\
\hline History of breastfeeding & \\
\hline Yes & \\
\hline Number of ANC visit & \\
\hline No ANC visit & \\
\hline$<4$ visits & \\
\hline$\geq 4$ visits & \\
\hline The numbers in the table indicate frequency (\%).
\end{tabular}


Two hundred forty-five (52.4\%) of the mothers had a high level of knowledge while 223 (47.6\%) had a low level of knowledge. From the variables included in the multiple linear regression model, i.e, age, education, employment, wealth tertile, number of children, and ANC visit, only maternal occupation significantly predicted breastfeeding knowledge score (Table 2). Compared to mothers who were housewives, mothers involved in small trades and private employment had significantly high level of breastfeeding knowledge score ( $\beta$ : $0.56 \mathrm{SD} ; 95 \% \mathrm{Cl}, 0.14,0.97 \mathrm{SD} ; P=0.009$ ). The mean \pm SD overall IIFAS score in our sample was $65.7 \pm 7.6$ points with range between 36 and 85 points. The majority $(60.9 \%)$ of the women had a neutral attitude towards breastfeeding whereas $36.9 \%$ of the participants had strongly positive attitudes toward breastfeeding. The variables that independently predicted maternal attitude towards breastfeeding included maternal age, education, employment and parity, household wealth status, and ANC visit (Table 3). Older mothers as compared to their younger counter parts (<20 years) $(\beta: 0.57 \mathrm{SD} ; 95 \% \mathrm{Cl}, 0.24$, $0.90 \mathrm{SD} ; P=0.001)$, and primiparous women as compared to multiparous $(\beta:-0.24 \mathrm{SD} ; 95 \% \mathrm{Cl},-0.47$, $-0.02 S D ; P=0.034$ ) had a significantly higher IIFAS score. Women who attended at least four ANC visits were found to have a significantly higher attitude towards breastfeeding than those who had no ANC visit ( $\beta$ : $0.41 \mathrm{SD} ; 95 \% \mathrm{Cl},(0.07,0.74 \mathrm{SD} ; P=0.017)$. we also found a statistically significant positive association between maternal BFKQ-AO and IIFAS scores ( $\beta$ : 0.33 SD; $95 \% \mathrm{Cl}, 0.25,0.41 \mathrm{SD} ; P<0.000$ ). 
Table 2

Predictors of BFKQ score among pregnant mothers in their second or third trimester $(N=468)$.

\begin{tabular}{|c|c|c|c|c|}
\hline Variable & Unadjusted $\beta$ (95\% Cl) & P-value & Adjusted $\beta(95 \% \mathrm{Cl})$ & P-value \\
\hline \multicolumn{5}{|l|}{ Age } \\
\hline $15-19$ & Ref & & Ref & \\
\hline $20-34$ & $0.16(-0.18,49.3)$ & 0.367 & $-0.19(-0.76,0.38)$ & 0.514 \\
\hline$\geq 35$ & $0.26(-0.23,75.3)$ & 0.291 & $-0.11(-0.78,0.56)$ & 0.753 \\
\hline \multicolumn{5}{|l|}{ Educational status } \\
\hline Illiterate & Ref & & Ref & \\
\hline Primary school & $-0.13(-0.39,0.13)$ & 0.335 & $-0.13(-0.42,0.16)$ & 0.389 \\
\hline Secondary school & $-0.00(-0.44,0.43)$ & 0.987 & $-0.03(-0.58,0.52)$ & 0.903 \\
\hline \multicolumn{5}{|l|}{ Maternal occupation } \\
\hline Housewife/Farmer & Ref & & Ref & \\
\hline Other & $0.68(0.31,1.06)$ & $<0.000^{\dagger}$ & $0.56(0.14,0.97)$ & $0.009^{\star}$ \\
\hline \multicolumn{5}{|l|}{ Wealth tertiles } \\
\hline Lowest & Ref & & Ref & \\
\hline Middle & $-0.19(-0.44,0.05)$ & 0.114 & $-0.05(-0.33,0.22)$ & 0.689 \\
\hline Highest & $-0.01(-0.220 .19)$ & 0.932 & $-0.07(-0.34,0.19)$ & 0.571 \\
\hline \multicolumn{5}{|l|}{ Parity } \\
\hline Primiparous & Ref & & Ref & \\
\hline Multiparous & $0.19(-0.06,0.45)$ & 0.139 & $0.69(-0.49,1.88)$ & 0.252 \\
\hline \multicolumn{5}{|l|}{ ANC visit } \\
\hline No ANC visit & Ref & & Ref & \\
\hline$<4$ visits & $-0.12(-0.35,0.11)$ & 0.324 & $-0.03(-0.29,0.23)$ & 0.813 \\
\hline$\geq 4$ visits & $0.18(-0.26,0.62)$ & 0.386 & $0.33(-0.18,0.85)$ & 0.203 \\
\hline \multicolumn{5}{|c|}{$t p<0.01$, significant values } \\
\hline$<0.05$, significé & & & & \\
\hline
\end{tabular}

Data are given as regression coefficients $(\beta)$ and $95 \%$ confidence interval. R2 is 0.05 .

BFKQ: Breastfeeding knowledge questionnaire, ANC: Antenatal Care 
Table 3

Predictors of IIFAS score among pregnant mothers in their second or third trimester $(\mathrm{N}=468)$.

\begin{tabular}{|c|c|c|c|c|}
\hline Variable & Unadjusted $\beta(95 \% \mathrm{Cl})$ & P-value & Adjusted $\beta$ (95\% Cl) & P-value \\
\hline \multicolumn{5}{|l|}{ Age } \\
\hline $15-19$ & Ref & & Ref & \\
\hline $20-34$ & $0.47(0.18,0.77)$ & $0.002^{\star}$ & $0.58(0.25,0.90)$ & $0.001^{*}$ \\
\hline$\geq 35$ & $0.46(-0.06,0.98)$ & 0.083 & $0.56(-0.02,1.13)$ & 0.060 \\
\hline \multicolumn{5}{|l|}{ Educational status } \\
\hline Illiterate & Ref & & Ref & \\
\hline Primary school & $-0.16(-0.41,0.09)$ & 0.207 & $-0.09(-0.32,0.14)$ & 0.433 \\
\hline Secondary school & $-0.00(-0.37,0.37)$ & 0.994 & $0.05(-0.33,0.42)$ & 0.813 \\
\hline \multicolumn{5}{|l|}{ Maternal occupation } \\
\hline Housewife/Farmer & Ref & & Ref & \\
\hline Other & $0.08(-0.31,0.46)$ & 0.702 & $-0.09(-0.46,0.26)$ & 0.593 \\
\hline \multicolumn{5}{|l|}{ Wealth tertiles } \\
\hline Lowest & Ref & & Ref & \\
\hline Middle & $0.08(-0.14,0.30)$ & 0.478 & $0.14(-0.08,0.35)$ & 0.217 \\
\hline Highest & $0.15(-0.08,0.38)$ & 0.206 & $0.15(-0.08,0.37)$ & 0.203 \\
\hline \multicolumn{5}{|l|}{ Parity } \\
\hline Primiparous & Ref & & Ref & \\
\hline Multiparous & $0.03(-0.18,0.25)$ & 0.778 & $-0.25(-0.48,-0.03)$ & $0.027^{*}$ \\
\hline \multicolumn{5}{|l|}{ ANC visit } \\
\hline No ANC visit & Ref & & Ref & \\
\hline$<4$ visits & $0.12(-0.17,0.40)$ & 0.430 & $0.16(-0.11,0.43)$ & 0.238 \\
\hline$\geq 4$ visits & $0.49(0.12,0.87)$ & $0.009 *$ & $0.41(0.08,0.75)$ & $0.017 *$ \\
\hline BFKQ score & $0.33(0.25,0.41)$ & $<0.000^{\dagger}$ & $0.33(0.25,0.41)$ & $<0.000^{\dagger}$ \\
\hline
\end{tabular}

Data are given as regression coefficients $(\beta)$ and $95 \%$ confidence interval. R2 is 0.15 . 
BFKQ: Breastfeeding knowledge questionnaire, IIFAS: lowa Infant Feeding Attitude Scale, ANC: Antenatal Care

\section{Discussion}

Breastfeeding is a choice made by all mothers, and this is highly affected by various factors including knowledge about and attitudes towards the benefits of breastfeeding. Despite Ethiopia attempts at increasing the rate of early initiation and duration of exclusive breastfeeding, the success is minimal. To the best of our knowledge, this is the first study assessing predictors of knowledge and attitude towards breastfeeding using validated instruments in Ethiopia. This community-based study revealed that half of the women had adequate knowledge about and the majority of the women had a neutral attitude toward breastfeeding. We also found that knowledge was associated only with maternal occupation while the women's attitude towards breastfeeding was associated with maternal age, parity, antenatal care visits and BFKQ-AO score.

In the present study, we found that half of the respondents had an adequate level of knowledge about breastfeeding. However, the presence of nearly half of women with a low level of knowledge demonstrates the importance of interventions that aim at improving the knowledge may be important in efforts to encourage women to breastfeed their child, and ultimately achieve the target set in the national health sector plan. Particularly, aspects of knowledge questions that scored lower percentage such as colostrum, breastmilk expression, the problem with breastfeeding and breast engorgement need emphasis during breastfeeding promotion.

Positive attitude toward breastfeeding is a strong predictor of breastfeeding initiation and duration than sociodemographic factors [19-25]. In this study, the mean IIFAS score was in a neutral attitude towards breastfeeding range [22]. The findings from the current study corroborate similar findings in the literature. Similar to the current study, a neutral attitude towards breastfeeding was reported across a diverse group of countries, China and Australia [16], Spain [32], Canada [33], Australia [34], Japan [35], and Scotland [20, 36]. Interestingly, most of these studies took place in Europe, and might not be comparable to our setting. However, it is possible that the finding of a neutral attitude could be one of the major reasons for a lower level of EBF in Ethiopia.

In the present study, women who were employed or merchants had higher knowledge about breastfeeding. This finding is similar to a study in China [17] reporting greater knowledge about breastfeeding among employed women. This is expected since maternal employment has been associated with education and those who are employed may be educated and thus have good knowledge about breastfeeding.

Earlier studies have shown that attitudes have been the focus of research because it is assumed that it always influence behavior; therefore, knowing something about a person's attitude can help us predict behavior in many contexts [37]. In this study, we found that younger women had higher attitude scores. In contrast, previous studies in China [16], Taiwan [38], Singapore [39], and Romania [40] showed that older 
mothers had more positive attitudes toward breastfeeding. The direct relation between higher IIFAS score (positive attitude) with increased age is likely linked to improved experience with breastfeeding. Breastfeeding is also in many aspects a learned behavior.

Maternal education is associated with favorable attitudes toward breastfeeding as highly educated women were more aware of breastfeeding benefits that help them develop a positive attitude. Though statistically not significant in the current study mothers who attended primary school had lower attitude score. Nevertheless, previous studies have shown that higher IIFAS-S scores were positively associated with a higher educational level in Lebanon [41], Ireland [42], China [17], Singapore [39] and Spain [32].

Studies report mixed results regarding the association between IIFAS score and occupation. A study from Ireland found that full or part-time employed mothers had more positive attitudes toward breastfeeding compared to housewives [42], while a study among Chinese women reported no significant difference between infant feeding attitudes and working status [16]. However, in our study, we found that employed or merchant mothers had more negative attitudes toward breastfeeding than housewives, but the difference was statistically not significant. It is conceivable that women who work may suffer from lack of time, fatigue, and experience breastfeeding as exhaustive leading to negative attitudes towards breastfeeding.

Higher maternal IIFAS score has been found by several other studies to be associated with higher family annual income in Lebanon [41], Taiwan [38], Singapore [39], Ireland [42] Spain [32]. In this study, we estimated household assets to generate a household asset score/wealth index since household income does not measure the value of non-monetary items, particularly in a low-income context. Nevertheless, we did not find that the IIFAS score was associated with the socio-economic status of the household in our setting.

Multiparous women are expected to have a positive attitude towards breastfeeding due to their prior experience, maternal confidence and ability to solve feeding problems. However, unlike findings from the Infant Feeding Survey where multiparous women had more favourable attitudes to breastfeeding [17], multiparous women in the current study had more negative attitudes towards breastfeeding. One can speculate that Ethiopian women with many children are taxed by household chores and limited time to breastfeed leading to negative attitudes. On the other hand, mothers with fewer children have more time and are more concerned with their newborn.

Pregnant women with $\geq 4$ antenatal care visits had higher IIFAS scores. A possible explanation might be that these women had increased exposure to prenatal breastfeeding education/counselling. Moreover, our respondents with high levels of breastfeeding knowledge also had a higher attitude score, in line with a finding from Finland [43]. This suggests that those mothers who have knowledge about breastfeeding also have a positive attitude.

This study has some limitations. Firstly, this is a cross-sectional study in design and thus we cannot establish causal relationships. Our findings are associations that may, or may not reflect cause and 
effect. Secondly, the majority of our participants had limited schooling, reducing the ability to detect a potentially statistically significant association between knowledge and attitude towards breastfeeding and educational status. The women in this study were homogeneous demographically and the findings may not be applicable across different regions of the country. Additional research is needed to assess the relationship between IIFAS and BFKQ and breastfeeding initiation and duration in urban Ethiopian settings.

\section{Conclusions}

We found that half of the respondents had adequate knowledge about breastfeeding; the majority of women had a neutral attitude towards breastfeeding. Occupation of the women was identified as an independent predictor of breastfeeding knowledge whereas age, antenatal care visit and BFKQ score were predictors of breastfeeding attitude. Thus, policymakers and managers should address these factors when planning educational interventions on breastfeeding to improve knowledge and attitude thereby improving breastfeeding practices in rural communities.

\section{Abbreviations}

BFESI: Breastfeeding Education and Support Intervention; BFKQ: Breastfeeding Knowledge Questionnaire; EBF: Exclusive breastfeeding; IIFAS: lowa Infant Feeding Attitudes Scale; ANC: Antenatal Care

\section{Declarations}

\section{Availability of data and materials}

The datasets used and/or analyzed during the current study are available from the corresponding author on reasonable request.

\section{Ethics approval and consent to participate}

The study protocol was approved by the Institutional Review Boards of the University of Oslo and Jimma University, and the ethical review board of Oromia Regional Health Bureau. Written consent of participation was obtained from eligible participants after they were provided with an information sheet.

\section{Consent for publication}

Not applicable

\section{Availability of data and materials}

The datasets used and/or analysed during the current study are available from the corresponding author on reasonable request. 


\section{Competing interests}

The authors declare that they have no competing interests.

\section{Funding}

This publication was supported by NORAD (Norwegian Agency for Development Cooperation) under the NORHED-Program, Agreement no. ETH-13/0024.

\section{Authors' contributions}

MAA, JHM and AF designed the research study. MAA performed the research. MAA and AA analysed the data and drafted the paper. All authors read and approved the final manuscript.

\section{Acknowledgements}

The authors would like to thank all the research participants who took part in the study.

\section{References}

1. Unicef: Global strategy for infant and young child feeding: World Health Organization; 2003.

2. Brown KH, Black RE, Lopez de Romana G, Creed de Kanashiro H: Infant-feeding practices and their relationship with diarrheal and other diseases in Huascar (Lima), Peru. Pediatrics 1989, 83(1):31-40.

3. Godfrey JR, Lawrence RA: Toward optimal health: the maternal benefits of breastfeeding. J Womens Health (Larchmt) 2010, 19(9):1597-1602.

4. Heinig MJ, Dewey KG: Health advantages of breast feeding for infants: a critical review. Nutrition research reviews 1996, 9(1):89-110.

5. Horta BL, Bahl R, Martinés JC, Victora CG, World Health O: Evidence on the long-term effects of breastfeeding : systematic review and meta-analyses. / Bernardo L. Horta ... [et al.]. In. Geneva: World Health Organization; 2007.

6. Popkin BM, Adair L, Akin JS, Black R, Briscoe J, Flieger W: Breast-feeding and diarrheal morbidity. Pediatrics 1990, 86(6):874-882.

7. Victora CG, Fuchs SC, Flores JAC, Fonseca W, Kirkwood B: Risk Factors for Pneumonia Among Children in a Brazilian Metropolitan Area. Pediatrics 1994, 93(6):977-985.

8. Arenz S, Ruckerl R, Koletzko B, von Kries R: Breast-feeding and childhood obesity-a systematic review. International journal of obesity and related metabolic disorders : journal of the International Association for the Study of Obesity 2004, 28(10):1247-1256.

9. Grummer-Strawn LM, Mei Z: Does breastfeeding protect against pediatric overweight? Analysis of longitudinal data from the Centers for Disease Control and Prevention Pediatric Nutrition Surveillance System. Pediatrics 2004, 113(2):e81-86. 
10. Harder T, Bergmann R, Kallischnigg G, Plagemann A: Duration of breastfeeding and risk of overweight: a meta-analysis. American journal of epidemiology 2005, 162(5):397-403.

11. Li R, Fein SB, Grummer-Strawn LM: Association of breastfeeding intensity and bottle-emptying behaviors at early infancy with infants' risk for excess weight at late infancy. Pediatrics 2008, 122 Suppl 2:S77-84.

12. Owen CG, Martin RM, Whincup PH, Smith GD, Cook DG: Effect of infant feeding on the risk of obesity across the life course: a quantitative review of published evidence. Pediatrics 2005, 115(5):13671377.

13. Victora CG, Bahl R, Barros AJ, França GV, Horton S, Krasevec J, Murch S, Sankar MJ, Walker N, Rollins NC: Breastfeeding in the 21st century: epidemiology, mechanisms, and lifelong effect. The Lancet 2016, 387(10017):475-490.

14. Central Statistical Agency (CSA) [Ethiopia] and ICF: Ethiopia demographic and health survey: key indicators report. In: Ethiopia, and Rockville, Maryland, USA CSA and ICF: Addis Ababa; 2016. 2016.

15. FDRE, MOH: Health Sector Transformation Plan 2015/16-2019/20. In.: Federal Democratic Republic of Ethiopia, Ministry of Health Addis Ababa ...; 2015.

16. Chen S, Binns CW, Liu Y, Maycock B, Zhao Y, Tang L: Attitudes towards breastfeeding - the lowa Infant Feeding Attitude Scale in Chinese mothers living in China and Australia. Asia Pacific journal of clinical nutrition 2013, 22(2):266-269.

17. Hamze L, Mao J, Reifsnider E: Knowledge and attitudes towards breastfeeding practices: A crosssectional survey of postnatal mothers in China. Midwifery 2019, 74:68-75.

18. Zhang Z, Zhu Y, Zhang L, Wan H: What factors influence exclusive breastfeeding based on the theory of planned behaviour. Midwifery 2018, 62:177-182.

19. Chen $\mathrm{C}-\mathrm{H}$, Chi C-S: Maternal intention and actual behavior in infant feeding at one month postpartum. Acta paediatrica taiwanica 2003, 44(3):140-144.

20. Dungy Cl, Mclnnes RJ, Tappin DM, Wallis AB, Oprescu F: Infant feeding attitudes and knowledge among socioeconomically disadvantaged women in Glasgow. Maternal and child health journal 2008, 12(3):313-322.

21. Jessri M, Farmer AP, Maximova K, Willows ND, Bell RC, Team AS: Predictors of exclusive breastfeeding: observations from the Alberta pregnancy outcomes and nutrition (APrON) study. BMC pediatrics $2013,13(1): 77$.

22. Mora Adl, Russell DW, Dungy Cl, Losch M, Dusdieker L: The lowa Infant Feeding Attitude Scale: Analysis of Reliability and Validity1. Journal of Applied Social Psychology 1999, 29(11):2362-2380.

23. Scott JA, Binns CW, Oddy WH, Graham KI: Predictors of breastfeeding duration: evidence from a cohort study. Pediatrics 2006, 117(4):e646-e655.

24. Sonko A, Worku A: Prevalence and predictors of exclusive breastfeeding for the first six months of life among women in Halaba special woreda, Southern Nations, Nationalities and Peoples' Region/SNNPR/, Ethiopia: a community based cross-sectional study. Archives of Public Health 2015, 73(1):53.

Page $14 / 16$ 
25. Thulier D, Mercer J: Variables associated with breastfeeding duration. Journal of Obstetric, Gynecologic \& Neonatal Nursing 2009, 38(3):259-268.

26. Bimerew A, Teshome M, Kassa GM: Prevalence of timely breastfeeding initiation and associated factors in Dembecha district, North West Ethiopia: a cross-sectional study. International Breastfeeding Journal 2016, 11.

27. Chekol DA, Biks GA, Gelaw YA, Melsew YA: Exclusive breastfeeding and mothers' employment status in Gondar town, Northwest Ethiopia: a comparative cross-sectional study. International Breastfeeding Journal 2017, 12(1):27.

28. Tsegaye M, Ajema D, Shiferaw S, Yirgu R: Level of exclusive breastfeeding practice in remote and pastoralist community, Aysaita woreda, Afar, Ethiopia. International Breastfeeding Journal 2019, 14(1):6.

29. Abdulahi M, Fretheim A, Magnus JH: Effect of breastfeeding education and support intervention (BFESI) versus routine care on timely initiation and exclusive breastfeeding in Southwest Ethiopia: study protocol for a cluster randomized controlled trial. BMC pediatrics 2018, 18:313.

30. Abdulahi M, Fretheim A, Argaw A, Magnus JH: Adaptation and validation of the lowa infant feeding attitude scale and the breastfeeding knowledge questionnaire for use in an Ethiopian setting. International Breastfeeding Journal 2020, 15(1):24.

31. Mora A, Russell D, Dungy C, Losch M, Dusdieker L: The lowa infant feeding attitude scale: analysis of reliability and validity. J App/ Soc Psychol 1999, 29.

32. Tomas-Almarcha R, Oliver-Roig A, Richart-Martinez M: Reliability and Validity of the Reduced Spanish Version of the lowa Infant Feeding Attitude Scale. Journal of obstetric, gynecologic, and neonatal nursing : JOGNN 2016, 45(5):e26-40.

33. Twells LK, Midodzi WK, Ludlow V, Murphy-Goodridge J, Burrage L, Gill N, Halfyard B, Schiff R, Newhook LA: Assessing Infant Feeding Attitudes of Expectant Women in a Provincial Population in Canada: Validation of the lowa Infant Feeding Attitude Scale. Journal of Human Lactation 2014, 32(3):NP9-NP18.

34. Cox KN, Giglia RC, Binns CW: The influence of infant feeding attitudes on breastfeeding duration: evidence from a cohort study in rural Western Australia. International Breastfeeding Journal 2015, 10(1):25.

35. Nanishi K, Jimba M: Reliability and Validity of the Japanese Version of the lowa Infant Feeding Attitude Scale: A Longitudinal Study. Journal of human lactation : official journal of International Lactation Consultant Association 2014, 30(3):346-352.

36. Scott JA, Shaker I, Reid M: Parental attitudes toward breastfeeding: their association with feeding outcome at hospital discharge. Birth (Berkeley, Calif) 2004, 31(2):125-131.

37. Ajzen I, Fishbein M: The Influence of Attitudes on Behavior. In. Volume 173, edn.; 2005: 173-221.

38. Ho YJ, McGrath JM: Predicting breastfeeding duration related to maternal attitudes in a taiwanese sample. The Journal of perinatal education 2011, 20(4):188-199. 
39. Lau Y, Htun TP, Lim Pl, Ho-Lim SS, Klainin-Yobas P: Psychometric Properties of the lowa Infant Feeding Attitude Scale among a Multiethnic Population during Pregnancy. Journal of human lactation : official journal of International Lactation Consultant Association 2016, 32(2):315-323.

40. Wallis AB, Brinzaniuc A, Chereches R, Oprescu F, Sirlincan E, David I, Dirle IA, Dungy Cl: Reliability and validity of the Romanian version of a scale to measure infant feeding attitudes and knowledge. Acta paediatrica (Oslo, Norway : 1992) 2008, 97(9):1194-1199.

41. Charafeddine L, Tamim H, Soubra M, de la Mora A, Nabulsi M: Validation of the Arabic Version of the lowa Infant Feeding Attitude Scale among Lebanese Women. Journal of human lactation : official journal of International Lactation Consultant Association 2016, 32(2):309-314.

42. Sittlington J, Stewart-Knox B, Wright M, Bradbury I, Scott JA: Infant-feeding attitudes of expectant mothers in Northern Ireland. Health education research 2007, 22(4):561-570.

43. Laanterä S, Pölkki T, Ekström A, Pietilä A-M: Breastfeeding attitudes of Finnish parents during pregnancy. BMC Pregnancy and Childbirth 2010, 10(1):79. 